\section{Umbilical cord stem cell therapy for bronchopulmonary dysplasia: ready for prime time?}

\author{
Christopher D Baker, Steven H Abman
}

Chronic lung diseases, such as chronic obstructive pulmonary disease (COPD), emphysema, idiopathic pulmonary fibrosis, cystic fibrosis, pulmonary arterial hypertension and others contribute significantly to morbidity and mortality worldwide, yet successful interventions are often limited. Over the past decade, there has been an explosion of excitement regarding potential roles for stem cell therapies to prevent, repair or regenerate lung tissue to improve outcomes of these severe, progressive and often fatal disorders. ${ }^{12}$ Based on strong preclinical data, early clinical studies are currently underway to explore safety and potential efficacy in adults with chronic pulmonary diseases, including COPD, idiopathic pulmonary arterial hypertension and others.

In children, bronchopulmonary dysplasia (BPD) remains one of the more common yet challenging respiratory disorders for which current strategies are extremely limited. BPD is the chronic lung disease that develops in premature newborns who require respiratory support and oxygen therapy after birth. ${ }^{3-7}$ BPD has changed remarkably since its original description over 45 years ago, and remains a 'moving target:' a disease modulated and at least partly defined by the impact of therapeutic interventions themselves. Routine use of prenatal steroids, surfactant therapy, improved ventilator strategies and other treatments have improved survival and the clinical course of infants with BPD. Importantly, infants with BPD now have very different clinical courses, outcomes and pathology than had been traditionally observed in preterm infants during the presurfactant era. The classic progressive stages leading to marked disruption of lung architecture with prominent fibroproliferative disease and cyst-like lesions that characterised BPD in the past are now often

\footnotetext{
Department of Pediatrics, Pediatric Heart Lung Center, University of Colorado School of Medicine and Children's Hospital Colorado, Aurora, Colorado, USA Correspondence to Dr Christopher D Baker, Department of Pediatrics, Pediatric Heart Lung Center, University of Colorado School of Medicine, $13123 \mathrm{E}$. 16th Ave Box B-395, Aurora, CO 80045, USA; Christopher.Baker@ucdenver.edu
}

absent, and the disease is now predominantly defined as a disruption of distal lung airspace and vascular growth. ${ }^{6-8}$ Lung histology of infants with the 'new BPD' often demonstrates far milder fibrosis and fewer signs of lung injury, but impaired alveolar and vascular growth remains prominent. ${ }^{8}$ Thus, changes in clinical, radiographic and histological features of the 'new BPD' reflect progressive incremental improvements in prenatal and postnatal management over the past several decades.

Despite these advances, BPD continues to be a major cause of neonatal morbidity and mortality. With increasing survival of the tiniest of premature babies, many infants still develop significant impairment of lung function, leading to late mortality, prolonged ventilator and neonatal intensive care unit (NICU) courses, frequent hospitalisations after NICU discharge, recurrent respiratory exacerbations, exercise intolerance, pulmonary hypertension and other signs of cardiorespiratory complications. $^{5-7}$ Some infants with BPD have late mortality and require tracheostomy and chronic mechanical ventilation throughout early childhood due to the severity of their lung disease. Recent studies further demonstrate that at least in some patients, marked abnormalities of lung function and decreased diffusion capacity cause significant morbidity that can persist into adulthood and may progress into advanced COPD. ${ }^{9}$

However, respiratory outcomes of infants with BPD can be widely variable and their clinical courses can be difficult to predict. Gestational age and the severity of prematurity are most strongly associated with high risk for BPD but diverse, interactive factors modulate the severity of disease. Postnatal mechanisms that contribute to the pathogenesis of BPD include oxidative and inflammation-mediated lung injury due to hyperoxia, infection, ventilator-induced injury and other adverse stimuli. Early and sustained inflammatory cells and cytokines in tracheal fluid from premature newborns are strongly associated with high risk for the subsequent development of BPD. ${ }^{10}$ Epidemiological and preclinical studies suggest that prenatal events, such as chorioamnionitis, pre-eclampsia, placental dysfunction and maternal smoking or substance abuse, may be sufficient to markedly increase the risk for BPD in preterm infants, perhaps independent of postnatal factors. It is likely that genetic, epigenetic and gene-environment interactions disrupt normal growth factor and cellular signalling during key stages of lung maturation, which impairs alveolar and vascular growth and results in the abnormal lung structure of BPD. ${ }^{6}$ Despite extensive studies, early biomarkers or clinical features to predict the severity of chronic lung disease in preterm infants are lacking. Some studies suggest that early interventions with antiinflammatory agents and strategies that directly preserve lung cell survival and function could prevent the development of BPD. Unfortunately, therapies are lacking that consistently and effectively attenuate lung injury, promote lung growth and lower the incidence or attenuate the severity of BPD.

Over the past several years, excitement has grown regarding the potential for progenitor or stem cell therapies as novel approaches to the prevention or treatment of BPD in preterm infants. ${ }^{11-13}$ Currently, specific mechanisms through which progenitor cells contribute to normal pulmonary development or protect the lung from injury remain unclear. Circulating endothelial progenitor cells (EPC) and mesenchymal stromal cells (MSC) are thought to contribute to lung development, but their physiological roles are unproven. Circulating early-outgrowth and late-outgrowth EPC can be isolated from peripheral blood and are thought to contribute to vascular development and repair after vascular injury. ${ }^{14} 15$ Rodents with experimental BPD due to hyperoxia have decreased EPC in the blood, lungs and bone marrow. ${ }^{16}$ Late-outgrowth endothelial colony forming cells (ECFC) are elevated in the cord blood of preterm human infants, but are decreased in those who go on to develop moderate-to-severe BPD. ${ }^{17} 18$ This suggests that disruption of ECFC-mediated vascular development may contribute to the pathobiology of BPD or merely provide a marker for BPD susceptibility.

Whereas the effects of ECFC in experimental BPD have not been fully explored, treatment with bone marrow-derived MSCs has shown promise in infant rats exposed to hyperoxia whether administered intravenously $^{19}$ or instilled directly into the trachea. $^{20}$ Each of these studies found minimal MSC engraftment in the lung, and that treatment with MSC-conditioned media (CdM, or 'the secretome') yielded similar benefit to MSC themselves. These findings suggested that MSC act to 
promote pulmonary vascular and alveolar development by paracrine mechanisms rather than cellular engraftment, as originally thought. Cord blood-derived MSCs are increased in extremely preterm infants, ${ }^{21}$ but associations with BPD severity and cord blood MSC number have not been shown. Increased MSC numbers from tracheal aspirates are associated with high risk for BPD and mortality, ${ }^{22}$ and importantly, these MSC secrete proinflammatory cytokines and differentiate into myofibroblasts that may promote lung injury. ${ }^{22-24}$ These findings seem disparate from studies that have demonstrated clear benefit of cord bloodderived MSCs in rodent models of BPD due to hyperoxia. Thus, MSCs isolated from various tissue compartments may be functionally distinct, and whether airway MSCs contribute to BPD pathogenesis or are a response to severe lung injury remains unclear.

Pierro et $a l^{25}$ report findings from their series of elegant and rigorous studies that clearly prove that early treatment of infant rats with human cord bloodderived MSC and umbilical cord perivascular cells or presumed pericytes (PC) can prevent the adverse effects of hyperoxia exposure on lung structure in infant rats. These authors also provide exciting new data that further demonstrate restoration of lung structure with late MSC and PC treatment. In addition, treatment with CdM from MSC and PC are sufficient for achieving similar effects on lung structure and function as observed with the cells themselves. These findings are wellsupported by comprehensive study endpoints that include improvements in lung structure, lung function, exercise test, pulmonary vascular growth and remodelling, and pulmonary hypertension with each therapy. There was no evidence of sustained cellular engraftment after cell therapies, and treated animals from each study group had no evidence of tumour formation by whole-body computerised tomography at 6 months.

Although past studies have shown potential efficacy of MSCs in prevention of BPD and acute lung injury, these data point to the novel clinical strategy of using cord derived cells, or MSC-like perivascular cells within the cord, or from MSCs harvested from cord blood itself. These exciting and novel findings demonstrate the potential for umbilical cordderived stem cell therapies for either the prevention or treatment of BPD.

Although these results are of great interest, the problem of how to begin to translate such strong preclinical findings into the clinical arena remains a very difficult challenge. Administration of umbilical cord-derived stem cells or their CdM resulted in effective treatment of neonatal hyperoxia, a common and useful rodent model of BPD. However, although this model includes histological and physiological features of human BPD, the clinical pathogenesis of BPD likely involves more complex stimuli reflecting an adverse prenatal environment and other postnatal factors beyond hyperoxia alone. In fact, several drug therapies have been shown to be at least as effective as MSC or MSC-derived CdM, including inhaled nitric oxide, vascular endothelial growth factor and sildenafil. ${ }^{26-28}$ Despite success in rodent models, for example, inhaled nitric oxide has had inconsistent effects in the prevention of BPD in the clinical setting. ${ }^{29-31}$ Given this uncertainty as to which therapies will effectively translate into the clinical setting, studies with preterm non-human primates and other large animals may help identify which modalities will be safe and effective for clinical trials.

Overall, strong preclinical data support the use of MSC or CdM from MSC for studies of human infants with BPD. However, many daunting questions persist regarding pragmatic aspects regarding the specific source of MSC for study, how to more clearly define MSC phenotype prior to administration, how to prepare cells for study and dose and mode of delivery. The potential for immunogenicity or risk for tumour formation suggests that cautious and prolonged safety monitoring will be essential.

Another critical question regarding the transition to clinical studies is how to select patients for study. We currently cannot readily predict or identify preterm infants who are at risk for severe BPD and sufficient biomarkers or other clinical predictors suggesting the need for early intervention are lacking. Alternatively, one can select only those infants with the most severe form of established BPD, but this is problematic due to marked variability among BPD infants regarding the impact of associated clinical problems, such as aspiration, tracheomalacia, airways reactivity, cardiac dysfunction and others.

Unlike the lung disease seen in small animal models, such as neonatal hyperoxia, the clinical setting of BPD is highly heterogeneous. BPD represents a clinical syndrome associated with diverse, multifactorial aetiologies, and includes infants with different phenotypes and a spectrum of outcomes, ranging from mild postnatal respiratory distress and essentially no long-term sequelae to those with severe life-threatening disease that, when survived, results in chronic respiratory failure and poor neurodevelopmental prognosis. Accounting for this variability in clinical trials is extremely difficult. Nevertheless, small trials, beginning with neonates with the most severe lung disease and poorest prognosis, are most likely to usher the most successful of these therapies into clinical practice. Finally, the rationale for translating these results to the clinical setting is strong, but how to approach the application of MSC therapy for the prevention of $B P D$ remains a considerable but worthwhile challenge.

\section{Competing interests None.}

Provenance and peer review Commissioned; internally peer reviewed.

To cite Baker CD, Abman SH. Thorax 2013;68:402-404.

Received 18 December 2012

Accepted 20 December 2012

Published Online First 15 January 2013

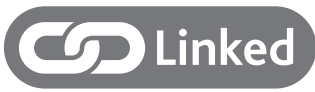

- http://dx.doi.org/10.1136/thoraxjnl-2012-202323

Thorax 2013;68:402-404.

doi:10.1136/thoraxjnl-2012-202661

\section{REFERENCES}

1 Beers MF, Morrisey EE. The three R's of lung health and disease: repair, remodeling and regeneration. J Clin Invest. 2011;121:2065-73.

2 Weiss DJ, Bertoncello I, Borok Z, et al. Stem cells and cell therapies in lung biology and lung diseases. Proc Am Thorac Soc 2011:8:223-72.

3 Northway WH Jr, Rosan RC, Porter DY. Pulmonary disease following respiratory therapy of hyaline membrane disease. N Engl J Med 1967;276:357-68.

4 Jobe $A H$, Bancalari E. Bronchopulmonary dysplasia. Am J Respir Crit Care Med 2001;163:1723-9.

5 Jobe $A H$. The new BPD: an arrest of lung development. Pediatr Res 1999;46:641-3.

6 Abman SH. Bronchopulmonary dysplasia. New York, NY: Informa Healthcare, 2010.

7 Baraldi E, Filippone M. Chronic lung disease after premature birth. N Engl J Med 2007;357:1946-55.

8 Hussain AN, Siddiqui NH, Stocker JT. Pathology of arrested acinar development in post-surfactant BPD. Hum Pathol 1998;29:710.

9 Wong PM, Lees AA, Louw J, et al. Emphsyema in young adult survivors of moderate to severe BPD. Eur Respir J. 2008;32:321-8.

10 Ambalavanan N, Carlo WA, D'Angio CT, et al. Cytokines associated with bronchopulmonary dysplasia or death in extremely low birth weight infants. Pediatrics 2009;123:1132-41.

11 Vosdoganes P, Lim R, Moss TJM, et al. Cell therapy: a novel treatment approach for BPD. Pediatrics 2012;130:1-11.

12 O'Reilly MO, Thebaud B. Cell based strategies to reconstitute lung function in infants with severe $B P D$. Clin Perinatol 2012;39:703-25.

13 Anversa P, Perrella MA, Kourembanas $S$, et al. Regenerative medicine: potential, promise, pitfalls and challenges. Eur J Clin Invest 2012;42:900-13.

14 Asahara T, Murohara T, Sullivan A, et al. Isolation of putative progenitor endothelial cells for angiogenesis. Science 1997;275:964-7. 
15 Ingram DA, Mead LE, Tanaka H, et al. Identification of a novel hierarchy of endothelial progenitor cells using human peripheral and umbilical cord blood. Blood 2004;104:2752-60.

16 Balasubramaniam V, Mervis CF, Maxey AM, et al. Hyperoxia reduces bone marrow, circulating, and lung endothelial progenitor cells in the developing lung: implications for the pathogenesis of bronchopulmonary dysplasia. Am J Physiol Lung Cell Mol Physiol 2007;292:L1073-84.

17 Baker C, Balasubramaniam V, Mourani P, et al. Cord blood angiogenic progenitor cells are decreased in bronchopulmonary dysplasia. Eur Respir J 2012; 40:1516-22.

18 Baker CD, Ryan SL, Ingram DA, et al. Endothelial colony-forming cells from preterm infants are increased and more susceptible to hyperoxia. Am J Respir Crit Care Med 2009;180: 454-61.

19 Aslam M, Baveja R, Liang OD, et al. Bone marrow stromal cells attenuate lung injury in a murine model of neonatal chronic lung disease. Am J Respir Crit Care Med 2009;180:1122-30.

20 Van Haaften T, Byrne R, Bonnet S, et al. Airway delivery of mesenchymal stem cells prevents arrested alveolar growth in neonatal lung injury in rats. Am J Respir Crit Care Med 2009;180:1131-42.

21 Javed MJ, Mead LE, Prater D, et al. Endothelial colony forming cells and mesenchymal stem cells are enriched at different gestational ages in human umbilical cord blood. Pediatr Res 2008;64:68-73.

22 Bozyk PD, Popova AP, Bentley JK, et al. Mesenchymal stromal cells from neonatal tracheal aspirates demonstrate a pattern of lung-specific gene expression. Stem Cells Dev 2011;20: 1995-2007.

23 Popova AP, Bozyk PD, Bentley JK, et al. Isolation of tracheal aspirate mesenchymal stromal cells predicts bronchopulmonary dysplasia. Pediatrics 2010;126: e1127-33.

24 Popova AP, Bozyk PD, Goldsmith AM, et al. Autocrine production of TGF-beta1 promotes myofibroblastic differentiation of neonatal lung mesenchymal stem cells. Am J Physiol Lung Cell Mol Physiol 2010;298:L735-43.

25 Pierro M, Ionescu L, Montemurro T, et al. Short-term, long-term and paracrine effect of human umbilical cord-derived stem cells in lung injury prevention and repair in experimental BPD. Thorax 2013;68: 475-84.
26 Kunig AM, Balasubramaniam V, Markham NE, et al. Recombinant human VEGF treatment enhances alveolarization after hyperoxic lung injury in neonatal rats. Am J Physiol Lung Cell Mol Physiol 2005;289: L529-35.

27 Ladha F, Bonnet S, Eaton F, et al. Sildenafil improves alveolar growth and pulmonary hypertension in hyperoxia-induced lung injury. Am J Respir Crit Care Med 2005;172:750-6.

28 Lin Y-J, Markham NE, Balasubramaniam V, et al. Inhaled nitric oxide enhances distal lung growth after exposure to hyperoxia in neonatal rats. Pediatr Res 2005;58:22-9.

29 Ballard RA, Truog WE, Cnaan A, et al. Inhaled nitric oxide in preterm infants undergoing mechanical ventilation. N Engl J Med 2006;355:343-53.

30 Kinsella JP, Cutter GR, Walsh WF, et al. Early inhaled nitric oxide therapy in premature newborns with respiratory failure. $N$ Engl J Med 2006;355:354-64.

31 Mercier JC, Hummler H, Durrmeyer $\mathrm{X}$, et al. Inhaled nitric oxide for prevention of bronchopulmonary dysplasia in premature babies (EUNO): a randomised controlled trial. Lancet 2010;376:346-54 